\title{
BMJ Open Use of the ICU Nurse-Physician Questionnaire (ICU N-P-Q): testing reliability and validity in neonatal intensive care units in Japan
}

\author{
Hatoko Sasaki, ${ }^{1,2,3}$ Naohiro Yonemoto, ${ }^{4}$ Rintaro Mori, ${ }^{2}$ Toshihiko Nishida, ${ }^{3}$ \\ Satoshi Kusuda, ${ }^{3}$ Takeo Nakayama ${ }^{1}$
}

To cite: Sasaki $\mathrm{H}$ Yonemoto N, Mori R, et al. Use of the ICU NursePhysician Questionnaire (ICU $\mathrm{N}-\mathrm{P}-\mathrm{Q}$ ): testing reliability and validity in neonatal intensive care units in Japan. BMJ Open 2016;6:e010105. doi:10.1136/bmjopen-2015010105

- Prepublication history and additional material is available. To view please visit the journal (http://dx.doi.org/ 10.1136/bmjopen-2015010105).

Received 26 September 2015 Revised 15 March 2016 Accepted 12 April 2016

CrossMark

For numbered affiliations see end of article.

Correspondence to

Hatoko Sasaki;

hatokos@hotmail.com

\section{ABSTRACT}

Objective: Although communication among health providers has become a critical part of improving quality of care, few studies on this topic have been conducted in Japan. This study aimed to examine the reliability and validity of the Intensive Care Unit Nurse-Physician Questionnaire (ICU N-P-Q) for use among nurses and physicians in neonatal ICUs (NICUs) in Japan.

Methods: A Japanese translation of the ICU N-P-Q was administered to physicians and nurses working at 40 NICUs across Japan, which were participating in the Improvement of NICU Practice and Team Approach Cluster randomized controlled trial (INTACT). We used the principal components analysis to evaluate the factor structure of the instruments. Convergent validity was assessed by examining correlations between the subscales of Communication and Conflict Management of the ICU N-P-Q and the subscales and total score of the Nurse-Physician Collaboration Scale (NPCS). Correlations between the subscales of Communication and Conflict Management by correlation with scales that refer to performance, including Job Satisfaction and Unit Effectiveness, were calculated to test the criterion validity.

Results: In total, 2006 questionnaires were completed by 316 physicians and 1690 nurses. The exploratory factor analysis revealed 15 factors in the physicians' questionnaire and 12 in the nurses' questionnaire. Convergent validity was confirmed, except for 'Between-group Accuracy' and 'Cooperativeness' in the physicians' scale, and for 'Between-group Accuracy' and 'Sharing of Patient Information' in the nurses' scale. Correlations between the subscales of communication and outcomes were confirmed in the nurses' questionnaire but were not fully supported in the physicians' questionnaire.

Conclusions: Although the psychometric property behaved somewhat differently by occupation, the present findings provide preliminary support for the utility of the common item structure with the original scale, to measure the degree and quality of communication and collaboration among staff at NICUs and similar healthcare settings in Japan.

Trial registration number: UMIN000007064; Pre-results.

\section{Strengths and limitations of this study}

- This is the first study to reveal the psychometric properties of the Intensive Care Unit NursePhysician Questionnaire (ICU N-P-Q) in a Japanese sample with a large number of working units.

- The present findings provided preliminary support for the Japanese ICU N-P-Q, which can be used to measure the extent and quality of communication/collaboration among medical and nursing staff at neonatal ICUs (NICUs) and similar healthcare settings in Japan.

- Examining the questionnaires for physicians and nurses separately may have revealed the psychometric properties more accurately than did the original study, which had a combined nursephysician sample.

- Some items were deleted from the questionnaire due to copyright restrictions. Therefore, the data in this study cannot fully compare with the psychometric property of the original study.

\section{INTRODUCTION}

Good relationships among staff in healthcare organisations are an essential factor to provide safe and high-quality care. Previous studies have observed that better communication and collaboration among healthcare providers is associated with higher technical quality of care, ${ }^{1}$ lower length of stay, ${ }^{2}$ superior clinical care in disease ${ }^{3}$ and risk-adjusted morbidity. ${ }^{4}$ Communication and collaboration among health professionals have been shown to make an impact on patient outcomes. A Cochrane Database of systematic Reviews ${ }^{5}$ found that practice-based interprofessional collaboration interventions enhanced healthcare processes and outcomes; however, generalising the core components of interprofessional collaboration interventions and their effectiveness remains an ongoing challenge. 
The aspects of communication include the degree to which physicians or nurses can carry out discussions without fear of repercussions or misunderstanding, the degree to which they believe in the consistent accuracy of the information conveyed by others, and the degree to which patient care information is relayed promptly to the people who need to be informed. ${ }^{6}$ Collaboration can be defined as the process where nurses and physicians work together in the delivery of quality care, jointly contributing in a balanced relationship characterised by mutual trust. ${ }^{7}$ There is a great deal of overlap between communication and collaboration; as Shortell $e t a l^{8}$ described, collaboration involves open and timely communication, integration of individuals' varied work activities, and ensuring that all available expertise is brought together to support problem-solving and conflict resolution. To advance our understanding of the impact and effectiveness of communication and collaboration on patient outcomes, it is critical to accurately assess the degree and quality of communication and collaboration among health professionals. A recent systematic review of survey instruments for measuring teamwork in healthcare settings identified 36 scales which met the study criteria. ${ }^{9}$ Twelve of the 36 scales documented relationships between teamwork and objective outcomes of interest in peer-reviewed studies. ${ }^{9}$ Another systematic review ${ }^{10}$ of survey instruments for assessing collaboration in healthcare settings found five instruments that met the study criteria for psychometric validity. The Intensive Care Unit Nurse-Physician Questionnaire (ICU N-P-Q) ${ }^{8}$ was one of the two scales identified by both reviews as a useful valid scale for future research.

The ICU N-P-Q was originally developed using a large national sample to measure collaboration at the ICU level and organisational components that facilitate a collaborative clinical interaction. The scale has been used to assess perceptions of nurse-physician collaboration in critical and non-critical care in the USA ${ }^{11-14}$ and the UK. ${ }^{15}$ A part of the scale was also used to assess leadership, disagreements and authority within the context of a neonatal ICU (NICU). ${ }^{16}$ The biggest difference between an ICU and an NICU is the body size of patients. Medication dosages of neonatal patients depend on their weight, and a large NICU is likely to have a much wider variety of diagnoses as compared with a small NICU. The number of beds is slightly larger in NICUs than in ICUs in Japan. ${ }^{17}$ Therefore, interprofessional communication in NICUs could be different from general ICUs and other healthcare groups, even in Japan. In this study, we aimed to examine the reliability and validity of the translated ICU N-P-Q among nurses and physicians from NICUs across Japan.

\section{METHODS}

Translation process

Permission to use the ICU N-P-Q and create a Japanese version was obtained from the original authors.
A professional translator of Japanese translated the original English version into Japanese, after which a different professional translator conducted back translation of the scale. However, two components of the scale (workplace and facility safety scales/culture) were not translated or included because of copyright restrictions. In order to maintain quality control, the back translation was shared with Dr Stephen M Shortell, Principal Investigator of the original study. ${ }^{8}$ After two authors (HS and RM) assessed the expressions used in the Japanese ICU N-P-Q, a pretest was performed on 30 physicians and 124 nurses from three preintervention facilities, which were participating in a trial known as the Improvement of NICU Practice and Team Approach Cluster randomized controlled trial (INTACT). The pretest aimed to assess whether the Japanese ICU N-P-Q was appropriate and easily understandable for nursing and physician personnel. The Japanese ICU N-P-Q was finalised after some modifications were made to the wording in response to pretest feedback.

\section{Ethical considerations}

Participation in this study was voluntary and written consent was obtained from each participant. Anonymity and confidentiality of the data were assured to all participants.

\section{Sample and data}

In this study, we used baseline data from a questionnaire distributed to physicians and nurses working at 40 NICUs that were participating in INTACT and located in different areas of Japan. Questionnaires were distributed to 345 physicians and 1800 nurses. The unlinked anonymous survey was administered from December 2011 to March 2012. We excluded data from the analysis if there were missing values for any variables in the ICU N-P-Q and if all or almost all of the items in each subscale were scored with the same number (eg, scored ' 1 ' in all values).

\section{Instrument \\ ICU Nurse-Physician Questionnaire}

The original ICU N-P-Q is a 120-item scale derived from the Organizational Culture Inventory with response items ranked on a five-point Likert scale ranging from $1=$ strongly disagree to $5=$ strongly agree. ${ }^{8} \mathrm{~A}$ revised and shortened version of the instrument is also available as an 81-item scale. In this study, we used the shorter version. Although a separate test for reliability and validity has not been completed for the shorter version, the authors who developed the ICU N-P-Q believed that the shorter version was easier to administer and was therefore able to achieve better survey compliance while ensuring good validity and reliability. ${ }^{18}$ Two components of the scale (workplace and facility safety scales/culture) were excluded because of copyright restrictions. ${ }^{19}$ The subscales of the ICU N-P-Q consist of Leadership, Communication, Coordination, Conflict Management, 
Unit Effectiveness and Authority, and a single item on Job Satisfaction. The scale includes separate questionnaires for physicians and nurses. Shortell et $a l^{8}$ reported that Cronbach's $\alpha$ reliabilities ranged from 0.61 to 0.88 for subscales. Other researchers have reported reliabilities from 0.66 to $0.92 .^{11} 131420$

\section{Nurse-Physician Collaboration Scale (NPCS)}

The NPCS $^{21}$ was developed to measure collaboration between nurses and physicians in Japan. The questionnaire is a 27-item scale and consists of three subscales: Joint Participation in Care, Sharing of Patient Information and Cooperativeness. Participants rate how often they experience these positive work-related states using a seven-point Likert scale ranging from $1=$ 'never' to 7='always/every day'. Cronbach's $\alpha$ reliabilities for nurses' responses to the subscales ranged from 0.80 to 0.92, and that for physicians' responses ranged from 0.84 to 0.93 . Psychometric testing showed that the NPCS was reliable and valid with high internal consistency and the results for test-retest reliability were adequate. Similar to the ICU N-P-Q the NPCS focuses on nurses' and physicians' collaborative and problem-solving skills. ${ }^{21}$ In this study, the NPCS was administered to test convergent validity of the Japanese ICU N-P-Q.

\section{Conceptual framework}

The conceptual framework of this study was based on the analytic framework of managerial and organisational factors affecting ICU performance, which was developed by Shortell $e t a l^{8}$ This concept focuses on the identification of main managerial practices and organisational processes that might influence effective performance. The important consideration is that these practices and processes are under the control of managers. According to this theory, organisational culture, leadership, communication, coordination and problem-solving should be included in these practices and processes. Specifically, a complex environment, such as that observed in ICUs, requires effective teamwork. More open, accurate and timely communication, as well as more open collaborative problem-solving approaches, would produce more effective patient care and improve health providers' occupational satisfaction. ${ }^{4} 22$ 23 The ICU N-P-Q consists of the Leadership and Authority scales assessing organisational factors, Communication and Conflict Management scales measuring the degree and quality of communication and collaboration within and between groups, and Unit Effectiveness and Job Satisfaction scales indicating outcomes of communication and collaboration. This study mainly focused on validating the Communication and Conflict Management scales of the ICU N-P-Q.

\section{Statistical analysis}

All statistical analyses were undertaken in SPSS V.21.0 (IBM Corp, USA). The $p$ value of $\leq 0.05$ was considered statistically significant.
Item analysis and reliability

First, the normality of the distribution of the scores was checked for each item using means, SDs, and skewness and kurtosis, and then the corrected item-total correlations and corrected item-subscale Cronbach's $\alpha$ were calculated separately for the physicians' and nurses' scales of the ICU N-P-Q. Items with corrected item-total correlations $<0.3,{ }^{24}$ and items with corrected item-subscale Cronbach's $\alpha>0.8$ were identified for possible exclusion from the scale.

\section{Factor analysis}

An exploratory factor analysis was conducted using a maximum likelihood solution method with promax rotation. The latent root criterion was used to decide the number of factors extracted, and factors having eigenvalues greater than 1 were considered significant. The Kaiser-Meyer-Olkin (KMO) was applied to measure the strength of the relationship among variables. KMO values greater than 0.7 are acceptable and values between 0.8 and 0.9 indicate a strong relationship. ${ }^{25}$ Factor loadings $>0.4$ were retained. If an item loaded equally on two factors, we dropped the item from the scales. Finally, means, SDs and internal consistency of the items were calculated for the factors that result from factor analysis. We also calculated interfactor correlations.

\section{Validity}

Convergent validity of the Communication and Conflict Management scales of the N-P-Q was assessed by means of the scales and total score of the NPCS, in which items are thought to reflect the fundamental aspects of the nurse-physician relationships. The Communication and Conflict Management scales of the N-P-Q included 'Within-group Accuracy', 'Between-group Accuracy', 'Between-group Avoiding Conflict Strategy' and 'Between-group Problem-solving Conflict Strategy' because the NPCS only examines the relationships between physicians and nurses. We assumed that the NPCS would have a positive correlation with the Japanese ICU N-P-Q. We also tested the criterion validity of the Communication and Conflict Management scales by examining their correlation with scales that refer to performance, including Job Satisfaction and Unit Effectiveness.

\section{RESULTS}

\section{Description of sample}

A total of 2006 questionnaires were completed by 316 physicians (response rate $=92 \%$ ) and 1690 nurses (response rate $=94 \%$ ). After excluding missing values and values scored with the same numbers, 1762 questionnaires were used in the analysis, including those of 285 physicians and 1475 nurses. Of the 285 participating physicians, 57 (20\%) were head physicians, $200(70.2 \%)$ were physicians, $24(8.4 \%)$ were residents and there 
were 4 missing values. Of the 1475 participating nurses, $130(8.8 \%)$ were head nurses, 1328 (90.0\%) were nurses, $2(1.0 \%)$ were assistant nurses and there were 15 missing values $(1.0 \%)$. The highest number of practice years in one's own unit was 5-9 years for nurses and $<1$ year for physicians (table 1 ).

\section{Item analysis and reliability}

Sixteen items were identified for possible exclusion from the physicians' scale. These included 3 items with corrected item-total correlations $<0.3$ (number 1, 9 and 38 ), and 13 items with corrected item-subscale Cronbach's $\alpha$ $>0.8$ (number 1, 9, 12, 24, 44, 45, 48, 51, 53, 66, 67, 68 and 75). Similarly, 14 items were identified for possible exclusion from the nurses' scale. These included five items with corrected item-total correlations $<0.3$ (number 1, 4, 9, 12 and 38), and nine items with corrected item-subscale Cronbach's $\alpha>0.8$ (number 30, 31, $32,44,49,51,66,67$ and 68) (see online supplementary appendix 1). Three of the four items in the 'Between-group Communication Openness' were dropped due to Cronbach's $\alpha>0.8$, and therefore the remaining item (number 29) was deleted for the factor analysis.

\section{Factor analysis}

The factor analysis for the physicians' scale returned to 15 factors $(\mathrm{KMO}=0.83, \mathrm{p}<0.001)$ (see online supplementary appendix 2). These 16 factors explained $56.3 \%$ of the observed variance. Nine items were dropped because three of them loaded $<0.4$. The following items that originally belonged to separate subscales were

\begin{tabular}{lcc} 
Table 1 Sample characteristics & \\
\hline & $\begin{array}{l}\text { Physicians } \\
\text { (N=285) } \\
\text { n (\%) }\end{array}$ & $\begin{array}{l}\text { Nurses } \\
\mathbf{( N = 1 4 7 5 )} \\
\mathbf{n}(\%)\end{array}$ \\
\hline Sex & & \\
Male & $195(68.4)$ & $25(1.7)$ \\
Female & $87(30.5)$ & $1430(96.9)$ \\
Missing & $3(1.1)$ & $20(1.4)$ \\
Status & & \\
Head physician & $57(20.0)$ & - \\
Physician & $200(70.2)$ & - \\
Resident & $24(8.4)$ & - \\
Missing & $4(1.4)$ & - \\
Head nurse & - & $130(8.8)$ \\
Nurse & - & $1328(90.0)$ \\
Assistant nurse & - & $2(1.0)$ \\
Missing & - & $15(1.0)$ \\
Years of practice & & $281(19.0)$ \\
$<1$ & $79(27.7)$ & $330(22.4)$ \\
1-2 & $49(17.2)$ & $304(20.6)$ \\
$3-4$ & $55(19.3)$ & $336(22.8)$ \\
$5-9$ & $53(18.6)$ & $208(14.1)$ \\
$>10$ & $46(16.1)$ & $16(1.1)$ \\
Missing & $3(1.1)$ & \\
& &
\end{tabular}

combined into one factor: two items on 'Within-group Avoiding Conflict Strategy' and three items on 'Between-group Avoiding Conflict Strategy', two items on 'Within-group Problem-solving Conflict Strategy' and three items on 'Between-group Problem-solving Conflict Strategy', three items on 'Absolute Technical Quality of Care' and one item on 'Perceived Effectiveness at Meeting Family Member Needs', and three items on 'Nursing Director Budgeting Authority' and two items on 'Nursing Director Patient Care Authority'.

The factor analysis revealed 12 factors in the nurses' scale $(\mathrm{KMO}=0.88, \mathrm{p}<0.001)$ (see online supplementary appendix 3). The 12-factor solution accounted for $45.8 \%$ of the total variance. Nine items with factor loadings $<0.4$ were deleted. The following items that originally belonged to separate subscales were combined into one factor: three items on 'Within-group Avoiding Conflict Strategy' and three items on 'Between-group Avoiding Conflict Strategy'; three items on 'Within-group Problem-solving Conflict Strategy' and two items on 'Between-group Problem-solving Conflict Strategy'; one item on 'Perceived Effectiveness at Recruiting and Retaining Nurses', one item on 'Perceived Effectiveness at Recruiting and Retaining Physicians', two items on 'Absolute Technical Quality of Care', and one item on 'Perceived Effectiveness at Meeting Family Member Needs'. Other items of physicians' and nurses' scales had the same factor structures reported by the original study.

\section{Validity}

\section{Convergent and criterion validity}

Correlations of the Communication and Conflict Management subscales of the ICU N-P-Q with the subscales and total score of the nurse-physician collaboration scale (NPCS) have been shown in table 2. Since the factor solutions did not reveal clear within-groups and between-groups distinctions for 'Avoiding Conflict Strategy' and 'Problem-solving Conflict Strategy', these scales were not included in the correlation matrix. A positive correlation was observed between the physicians' scale and the NPCS, except for 'Between-group Accuracy' and 'Cooperativeness' ( $\mathrm{r}=0.081, \mathrm{p}=0.173)$. Similarly, a positive correlation was observed between the nurses' scale and the NPCS, except for 'Between-group Accuracy' and 'Sharing of Patient Information' ( $\mathrm{r}=0.036, \mathrm{p}=0.162)$.

The correlations between the subscales on communication/collaboration (Communication, Coordination and Conflict Management) and the subscales on performance (Job Satisfaction and Unit Effectiveness) in the ICU N-P-Q have been shown in table 3. Positive correlations were observed for the physicians' subscales, except for 'Within-group Openness' and 'Perceived Effectiveness at Recruiting and Retaining Nurses' $(\mathrm{r}=0.096, \mathrm{p}=0.11)$. There were positive correlations for all the subscales of the nurses' scale. 
Table 2 Correlation coefficients (Pearson $r$ ) for the subscales on communication/collaboration of the ICU Nurse-Physician Questionnaire with the subscales and total score of the NPCS

\begin{tabular}{|c|c|c|c|c|c|c|c|c|c|c|c|c|c|c|c|c|c|}
\hline & \multirow[b]{4}{*}{ Subscales } & \multicolumn{16}{|l|}{ NPCS } \\
\hline & & \multicolumn{4}{|c|}{ Joint Participation in Care } & \multicolumn{4}{|c|}{ Sharing of Patient Information } & \multicolumn{4}{|c|}{ Cooperativeness } & \multicolumn{4}{|l|}{ Total } \\
\hline & & \multicolumn{2}{|l|}{ Doctor } & \multicolumn{2}{|l|}{ Nurse } & \multicolumn{2}{|l|}{ Doctor } & \multicolumn{2}{|l|}{ Nurse } & \multicolumn{2}{|l|}{ Doctor } & \multicolumn{2}{|l|}{ Nurse } & \multicolumn{2}{|l|}{ Doctor } & \multicolumn{2}{|l|}{ Nurse } \\
\hline & & Correlation & $\begin{array}{l}p \\
\text { Value }\end{array}$ & Correlation & $\begin{array}{l}\mathbf{p} \\
\text { Value }\end{array}$ & Correlation & $\begin{array}{l}p \\
\text { Value }\end{array}$ & Correlation & $\begin{array}{l}p \\
\text { Value }\end{array}$ & Correlation & $\begin{array}{l}\mathbf{p} \\
\text { Value }\end{array}$ & Correlation & $\begin{array}{l}p \\
\text { Value }\end{array}$ & Correlation & $\begin{array}{l}p \\
\text { Value }\end{array}$ & Correlation & $\begin{array}{l}p \\
\text { Value }\end{array}$ \\
\hline $\begin{array}{l}\text { ICU Nurse- } \\
\text { Physician }\end{array}$ & $\begin{array}{l}\text { Between-group } \\
\text { Openness }\end{array}$ & 0.270 & $<0.001$ & NA & & 0.248 & $<0.001$ & NA & & 0.525 & $<0.001$ & NA & & 0.402 & $<0.001$ & NA & \\
\hline Questionnaire & $\begin{array}{l}\text { Between-group } \\
\text { Accuracy }\end{array}$ & 0.224 & $<0.001$ & 0.154 & $<0.001$ & 0.117 & $<0.048$ & 0.036 & 0.162 & 0.080 & 0.173 & 0.073 & $<0.005$ & 0.155 & $<0.009$ & 0.098 & $<0.001$ \\
\hline
\end{tabular}

ICU, intensive care unit; NA, not applicable; NPCS, Nurse-Physician Collaboration Scale.

Table 3 Correlations between the subscales on communication/collaboration and the outcomes

\begin{tabular}{|c|c|c|c|c|c|c|c|c|c|c|c|c|c|c|c|c|}
\hline & & & \multicolumn{14}{|c|}{ Subscales of Communication/Collaboration } \\
\hline & & & \multicolumn{2}{|c|}{$\begin{array}{l}\text { Unit Relations with } \\
\text { Other Units }\end{array}$} & \multicolumn{2}{|c|}{$\begin{array}{l}\text { Within-group } \\
\text { Openness }\end{array}$} & \multicolumn{2}{|l|}{$\begin{array}{l}\text { Within-group } \\
\text { Accuracy }\end{array}$} & \multicolumn{2}{|c|}{$\begin{array}{l}\text { Between-group } \\
\text { Openness }\end{array}$} & \multicolumn{2}{|c|}{$\begin{array}{l}\text { Between-group } \\
\text { Accuracy }\end{array}$} & \multicolumn{2}{|c|}{ Avoiding Conflict } & \multicolumn{2}{|c|}{$\begin{array}{l}\text { Problem-solving } \\
\text { Conflict }\end{array}$} \\
\hline & & & Correlation & $\begin{array}{l}\mathbf{p} \\
\text { Value }\end{array}$ & Correlation & $\begin{array}{l}\mathbf{p} \\
\text { Value }\end{array}$ & Correlation & $\begin{array}{l}p \\
\text { Value }\end{array}$ & Correlation & $\begin{array}{l}p \\
\text { Value }\end{array}$ & Correlation & $\begin{array}{l}p \\
\text { Value }\end{array}$ & Correlation & $\begin{array}{l}p \\
\text { Value }\end{array}$ & Correlation & $\begin{array}{l}p \\
\text { Value }\end{array}$ \\
\hline \multirow{6}{*}{$\begin{array}{l}\text { Subscales of } \\
\text { Outcome }\end{array}$} & \multirow{2}{*}{$\begin{array}{l}\text { Perceived effectiveness } \\
\text { nurses }\end{array}$} & Doctor & 0.162 & $<0.006$ & 0.096 & 0.105 & 0.202 & $<0.001$ & 0.155 & $<0.009$ & 0.256 & $<0.001$ & 0.216 & $<0.001$ & 0.257 & $<0.001$ \\
\hline & & Nurse & 0.225 & $<0.001$ & 0.107 & $<0.001$ & 0.148 & $<0.001$ & NA & & 0.115 & $<0.001$ & 0.183 & $<0.001$ & 0.230 & $<0.001$ \\
\hline & \multirow{4}{*}{$\begin{array}{l}\text { Absolute technical quality of } \\
\text { care/effectiveness at meeting } \\
\text { family member needs } \\
\text { Satisfaction }\end{array}$} & Doctor & 0.228 & $<0.001$ & 0.341 & $<0.001$ & 0.325 & $<0.001$ & 0.168 & $<0.004$ & 0.261 & $<0.001$ & 0.263 & $<0.001$ & 0.444 & $<0.001$ \\
\hline & & Nurse & 0.318 & $<0.001$ & 0.207 & $<0.001$ & 0.243 & $<0.001$ & NA & & 0.214 & $<0.001$ & 0.298 & $<0.001$ & 0.432 & $<0.001$ \\
\hline & & Doctor & 0.231 & $<0.001$ & 0.395 & $<0.001$ & 0.250 & $<0.001$ & 0.192 & $<0.001$ & 0.117 & $<0.052$ & 0.192 & $<0.001$ & 0.343 & $<0.001$ \\
\hline & & Nurse & 0.324 & $<0.001$ & 0.440 & $<0.001$ & 0.198 & $<0.001$ & NA & & 0.106 & $<0.001$ & 0.230 & $<0.001$ & 0.276 & $<0.001$ \\
\hline
\end{tabular}




\section{Description of the scales}

The lowest score was given for 'Between-group Communication Accuracy' (physician: mean=2.86, $\mathrm{SD}=0.73$ ) and 'Perceived Effectiveness at Recruiting and Retaining' (nurse: mean $=3.00, \mathrm{SD}=0.56$ ). The highest scores were given for 'Within-group Communication Openness' (physician: mean $=3.95, \quad \mathrm{SD}=0.71$ ) and 'Avoiding Conflict Strategy' (nurse: mean=3.70, SD=0.60). Almost all of the subscales demonstrated good to high reliability for physicians ranged from 0.54 to 0.89 and for nurses ranged from 0.51 to 0.87 . The lowest $\alpha$ value was found in 'Perceived Effectiveness at Recruiting and Retaining' for physicians with 0.54 and for nurses with 0.51 . The interfactor correlation ranged from -0.03 to 0.58 in physicians and from -0.01 to 0.54 in nurses. Negative interfactor correlations were found between Factor 1 and Factor 13, Factor 3 and Factor 13, Factor 4 and Factor 13, Factor 7 and Factor 13, Factor 11 and Factor 13, and Factor 12 and Factor 13 for physicians. Interfactor correlation between Factor 1 and Factor 11, and between Factor 11 and Factor 12, was negative correlation for nurses (see online supplementary appendices 4 and 5).

\section{DISCUSSION}

Main findings

This is the first study to reveal the psychometric property of the ICU N-P-Q in a Japanese sample with a large number of working units. Fifteen of the 21 scales for physicians, and 12 of the 21 scales for nurses, were retained as a result of the factor analysis. The factor structure and interfactor correlations were in the theoretically unexpected directions for both scales, where there was no distinction between the within-group and between-group factor solutions on 'Avoiding Conflict Strategy' and 'Problem-solving Conflict Strategy'. Convergent validity was confirmed by assessing correlations between the NPCS and the Communication and Conflict Management subscales of the ICU N-P-Q except for 'Between-group Accuracy' and 'Cooperativeness' from the physicians' scale and 'Between-group Accuracy' and 'Sharing of Patient Information' from the nurses' scale. With reference to concurrent validity, the predicted relationships between the subscales of communication and outcomes were confirmed in the nurses' questionnaire but were not fully supported in the physicians' questionnaire.

\section{Explanation and interpretation}

The number of factors in the physicians' scale was not identical with that in the nurses' scale, where the 'Absolute Technical Quality of Care' was combined with 'Perceived Effectiveness at Meeting Family Member Needs' in both scales. This suggests that the items in these two subscales may not group well. There was no distinction between the within-group and between-group factor solutions on 'Avoiding Conflict Strategy' and
'Problem-solving Conflict Strategy'. This may be because the conflicts between nurses and physicians are due to the overlapping nature of their domains and the lack of clarity regarding their roles ${ }^{26}$ and they differ in terms of their beliefs about responsibility, barriers to progress and possible solutions to the problem. ${ }^{27}$ In some NICUs, nurses indeed fulfil a part of the physicians' role in Japan.

'Cooperativeness' in the NPCS did not correlate with the 'Between-group Accuracy' of the ICU N-P-Q for physicians, while 'Sharing of Patient Information' in the NPCS did not associate with the 'Between-group Accuracy' of the ICU N-P-Q for nurses. Although there are correlations between 'Cooperativeness' and 'Between-group Accuracy' for nurses, and 'Sharing of Patient Information' and 'Between-group Accuracy' for physicians, these correlations are weak. 'Cooperativeness' and 'Sharing of Patient Information' in the NPCS may not have reflected concepts similar to the 'Between-group Accuracy' subscale in the ICU N-P-Q.

Although Cronbach's $\alpha$ coefficients for the nurses' and physicians' questionnaires were mostly acceptable, they were not fully comparable with the original validation study ${ }^{8}$ and previous studies using the ICU N-P-Q ${ }^{11-14}$ which had a combined nurse-physician sample. The lowest reliability was found in the subscale 'Perceived Effectiveness at Recruiting and Retaining' for both questionnaires. To enhance the subscale's consistency, the items could be refined by several additional statements. It is important to consider these aspects when administering the scale.

Two issues need to be examined in future studies. First, the construct validity of the original English version needs to be examined more closely because though the ICU N-P-Q is one of the well-known measures on the organisational culture and communication in healthcare settings ${ }^{10}$ the questionnaire has been used only partially. ${ }^{4} 11 \quad 12 \quad 19$ This also restricts comparison across studies and countries. Second, the findings of the present study revealed that several subscales are different constructs of the original scales. We did not rename or eliminate these subscales in this study because further validity would clarify why several subscales that originally belonged to separate scales were combined in this study, and how these can be distinct constructs.

This study examined the questionnaires for physicians and nurses separately. Therefore, the present results may have revealed the psychometric properties more accurately than did the original study, which had a combined nurse-physician sample, and highlighted some points for further research concerning the difference between perceptions of physicians and nurses. Considering the burden of administration time and the response rate to the short version of the 81 -item scale, it might be a better approach to use only selected parts of the scales depending on the purpose of individual studies and researchers' specific interests, as previous studies have done. $^{11-1319}$ 


\section{Limitations}

This present study has a few limitations. First, two components (workplace and facility safety scales/culture) of the original instrument were not available because of copyright restrictions. Second, some items and subscales (eg, 'Team Cohesion', 'Understanding', 'Satisfaction with Nurse Communication', 'Satisfaction with Physician Communication', 'Within-group Forcing', 'Betweengroup Forcing', 'Within-group Arbitration' and 'Betweengroup Arbitration') were not included in the shorter version of the physician and nurse questionnaires. Therefore, the data in this study cannot fully compare with the psychometric property of the original study. Third, the NPCS measures the cooperation between physicians and nurses, and therefore examination of the scale correlations only with the two subscales assessing openness and accuracy between groups was appropriate for testing the convergent validity. Finally, this study could not determine whether the differences in the factorial structure are caused by the sample characteristics or cultural differences, since the original study did not perform an item analysis or factor analysis.

\section{CONCLUSION}

Although the psychometric property of the Japanese ICU N-P-Q acted slightly differently in this study according to occupation, the present findings provide preliminary support for the utility of the common item structure of the original scale to measure the extent and quality of communication and collaboration among medical and nursing staff at NICUs and similar healthcare settings in Japan.

\author{
Author affiliations \\ ${ }^{1}$ Department of Health Informatics, School of Public Health, Kyoto University, \\ Kyoto, Japan \\ ${ }^{2}$ Department of Health Policy, National Center for Child Health and \\ Development, Tokyo, Japan \\ ${ }^{3}$ Department of Neonatology, Maternal and Perinatal Center, Tokyo Women's \\ Medical University, Tokyo, Japan \\ ${ }^{4}$ Department of Biostatistics, Kyoto University School of Public Health, Kyoto, \\ Japan
}

Acknowledgements The authors wish to thank Dr Atsushi Uchiyama and Dr Hideko Mitsuhashi (Tokyo Women's Medical University), and all physicians and nurses who generously participated in this study. They also thank Ms Emma Barber (National Center for Child Health and Development) for her editorial support.

Contributors HS administered the survey, acquired the data, performed the statistical analysis, and prepared the draft. NY provided supervision of the study design, the data analysis and interpretation. RM supervised the design of the study. TNi and SK managed the whole research process. TNa supervised the data analysis and critically revised the manuscript for important intellectual content. All authors were involved in the critical commentary and approved the final version of the manuscript.

Funding Health and Labour Sciences Research Grants in FY2012 (H23-Iryo Shitei-008) were funded by the Ministry of Health, Labour and Welfare, Japan.

Disclaimer The funder had no role in the study design, data collection and analysis, decision to publish, or preparation of the manuscript.

Competing interests None declared.
Ethics approval Ethical approval was obtained on 15 July 2011 from the independent review board of Improvement of NICU Practice and Team Approach Cluster randomized controlled trial (INTACT: UMIN000007064), which has its administrative office in Tokyo Women's Medical University. This study was also approved by the Ethics Committee of the Kyoto University Graduate School and Faculty of Medicine on 28 March 2014.

Provenance and peer review Not commissioned; externally peer reviewed.

Data sharing statement No additional data are available.

Open Access This is an Open Access article distributed in accordance with the Creative Commons Attribution Non Commercial (CC BY-NC 4.0) license, which permits others to distribute, remix, adapt, build upon this work noncommercially, and license their derivative works on different terms, provided the original work is properly cited and the use is non-commercial. See: http:// creativecommons.org/licenses/by-nc/4.0/

\section{REFERENCES}

1. Shortell SM, Zimmerman JE, Rousseau DM, et al. The performance of intensive care units: does good management make a difference? Med Care 1994;32:508-25.

2. Narasimhan M, Eisen LA, Mahoney CD, et al. Improving nurse-physician communication and satisfaction in the intensive care unit with a daily goals worksheet. Am J Crit Care 2006;15:217-22.

3. Bower $\mathrm{P}$, Campbell $\mathrm{S}$, Bojke $\mathrm{C}$, et al. Team structure, team climate and the quality of care in primary care: an observational study. Qual Saf Health Care 2003;12:273-9.

4. Davenport DL, Henderson WG, Mosca CL, et al. Risk-adjusted morbidity in teaching hospitals correlates with reported levels of communication and collaboration on surgical teams but not with scale measures of teamwork climate, safety climate, or working conditions. J Am Coll Surg 2007;205:778-84.

5. Zwarenstein M, Goldman J, Reeves S. Interprofessiona collaboration: effects of practice-based interventions on professional practice and healthcare outcomes. Cochrane Database Syst Rev 2009;(3):CD000072.

6. Roberts $\mathrm{KH}$, O'Reilly CA. Measuring organizational communication. J Appl Psychol 1974;59:321.

7. Alt-White AC, Charns M, Strayer R. Personal, organizational and managerial factors related to nurse-physician collaboration. Nurs Adm Q 1983;8:8-18.

8. Shortell SM, Rousseau DM, Gillies RR, et al. Organizational assessment in intensive care units (ICUs): construct development, reliability, and validity of the ICU Nurse-Physician Questionnaire. Med Care 1991;29:709-26.

9. Valentine MA, Nembhard IM, Edmondson AC. Measuring teamwork in health care settings: a review of survey instruments. Med Care 2015;53:e16-30.

10. Dougherty $\mathrm{MB}$, Larson $\mathrm{E}$. A review of instruments measuring nurse-physician collaboration. J Nurs Adm 2005;35:244-53.

11. Manojlovich M, DeCicco B. Healthy work environments, nurse-physician communication, and patients' outcomes. Am J Crit Care 2007;16:536-43.

12. Miller PA. Nurse-physician collaboration in an intensive care unit. Am J Crit Care 2001;10:341-50.

13. Manojlovich M, Antonakos CL, Ronis DL. Intensive care units, communication between nurses and physicians, and patients' outcomes. Am J Crit Care 2009;18:21-30.

14. Manojlovich M. Linking the practice environment to nurses' job satisfaction through nurse-physician communication. J Nurs Scholarsh 2005;37:367-73.

15. Reader TW, Flin R, Mearns K, et al. Interdisciplinary communication in the intensive care unit. Br J Anaesth 2007;98:347-52.

16. Baker GR, King H, MacDonald JL, et al. Using organizational assessment surveys for improvement in neonatal intensive care. Pediatrics 2003;111(Suppl E1):e419-25.

17. Hospital Accreditation Standards Databook. Japan Council for Quality Health Care. 2010. http://jcqhc.or.jp/pdf/research/databook_ h21.pdf

18. Excerpted from The Organization and Management of Intensive Care Units. Copyright 1989, Shortell and Rousseau. http:// shortellresearch.berkeley.edu/ICU\%20Questionnaires.htm

19. Cooke RA LJ. Level V: organizational culture inventory. Plymouth, Michigan: Human Synergistics, 1987.

20. Hansen HE, Biros MH, Delaney NM, et al. Research utilization and interdisciplinary collaboration in emergency care. Acad Emerg Med 1999;6:271-9. 
21. Ushiro R. Nurse-Physician Collaboration Scale: development and psychometric testing. J Adv Nurs 2009;65: 1497-508.

22. Blegen MA. Nurses' job satisfaction: a meta-analysis of related variables. Nurs Res 1993;42:36-41.

23. Pesudovs K, Garamendi E, Keeves JP, et al. The activities of daily vision scale for cataract surgery outcomes: re-evaluating validity with Rasch analysis. Invest Ophthalmol Vis Sci 2003;44:2892-9.

24. Field A. Discovering statistics using SPSS. Sage Publications, 2009
25. Hutcheson GD, Sofroniou N. The multivariate social scientist: introductory statistics using generalized linear models. Sage, 1999.

26. Weiss SJ. Role differentiation between nurse and physician: implications for nursing. Nurs Res 1983;32:133-9.

27. Rosenstein $\mathrm{AH}, \mathrm{O}$ 'Daniel M. Original research: disruptive behavior and clinical outcomes: perceptions of nurses and physicians: nurses, physicians, and administrators say that clinicians' disruptive behavior has negative effects on clinical outcomes. Am J Nurs 2005;105:54-64. 